\title{
Intracerebellar Schwannoma in Presumed Schwannomatosis: A Case Report
} \author{
Korea \\ Corresponding author: \\ Jeong Hoon Kim \\ Department of Neurological Surgery, \\ Asan Medical Center, University of \\ Ulsan College of Medicine, 88, \\ Olympic-ro 43-gil, Songpa-gu, Seoul \\ 05505, Republic of Korea \\ Tel: + $82-2-3010-3550$ \\ Fax: +82-2-476-6738 \\ E-mail: jhkim1@amc.seoul.kr
}

Myeongjong Kim, Jeong Hoon Kim

Department of Neurological Surgery, Asan Medical Center, University of Ulsan College of Medicine, Seoul, Republic of

Received: March 1, 2018

Revised: March 14, 2018

Accepted: March 15, 2018

\begin{abstract}
It is generally believed that schwannomas are derived from the nerve sheath. Intracerebellar schwannomas are rare, especially in patients with schwannomatosis. In this report, we present a case of intracerebellar schwannoma in presumed schwannomatosis. A 31-year-old man presented with headache and vomiting; he then underwent computed tomography, magnetic resonance imaging (MRI), and transfemoral cerebral angiography. MRI revealed an intracerebellar tumor and multiple spinal cord tumors. The brain tumor was removed completely through midline suboccipital craniotomy. The histological diagnosis of schwannoma was confirmed. The patient was not tested for mutation in the neurofibromatosis gene. However, all his symptoms were consistent with a diagnosis of schwannomatosis. Intracerebellar schwannomas are rare tumors; however, they should be considered as differential diagnoses when a well-circumscribed nodule in the cerebellar hemisphere with typical signs of schwannoma is present in patients with schwannomatosis.
\end{abstract}

Key Words: Cerebellar neoplasms; Neurilemmoma; Schwannomatosis

\section{INTRODUCTION}

Schwannomatosis is a form of neurofibromatosis $(N F)$ that is clinically and genetically distinct from NF type 1 (NF1) and $\mathrm{NF}$ type 2 (NF2). It is defined as the presence of two or more non-intradermal schwannomas, at least one with pathological confirmation, including absence of bilateral vestibular schwannoma on high-quality brain magnetic resonance imaging (MRI) and no known $N F$ mutation ${ }^{4)}$. Schwannomatosis is rare disease, having an annual incidence of 0.58 cases per 1,000,000 persons, with approximately $20 \%$ of cases being familial ${ }^{1)}$.

It is commonly believed that schwannomas are derived from the nerve sheath. Cases of intraparenchymally located schwannomas in the central nervous system are very limited ${ }^{2,-9)}$. Moreover, intracerebellar schwannoma in patients with schwannomatosis is extremely rare. We present a case of intracerebellar schwannoma verified by pathological diagnosis in a patient with presumed schwannomatosis.

\section{CASE REPORT}

A 31-year-old man was admitted to our neurosurgical department with a 6-month history of progressive headache and vomiting. He was neurologically intact, and there were no cutaneous or skeletal signs of $\mathrm{NF}$. He did not have any family history of NF and schwannomatosis.

Brain computed tomography (CT) revealed a low-attenuation and round-shaped lesion in the right cerebellum. MRI revealed a round-shaped lesion in the right cerebellum with a clear margin and low signal intensity on T1-weighted imaging (WI; Fig. 1A), and non-uniform, high signal intensity on T2-WI (Fig. 1B). In contrast-enhanced MRI, a homogeneous, enhanced solid portion and a non-enhanced cystic portion were verified (Fig. 1C, D). Compression of the fourth ventricle and brain stem, and mild hydrocephalus were identified. In whole-spine MRI, multiple well-enhanced intradural extramedullary nodular lesions involving levels $\mathrm{C} 1$ to $\mathrm{C} 3, \mathrm{C} 5$ to $\mathrm{C} 6$, T6, and $\mathrm{L} 2$ to $\mathrm{L} 5$ were verified (Fig. 2A, B). Chest and abdominopelvic CT were performed and there was no evidence of a primary tumor or metastasis. The differential diagnosis was ependymoma with leptomeningeal metastasis versus multiple hemangioblastomas. Transfemoral cerebral angiography was performed and there was no definite tumor staining.

Because of hydrocephalus with brain tumor, endoscopic third ventriculostomy was initially performed, followed by midline suboccipital craniotomy and removal of the tumor. During surgery, we found that the tumor was located under the right cerebellar hemisphere cortex and had no obvious relationship with the surrounding dura and cranial nerve. The postoperative course was uneventful. His headache had re- 

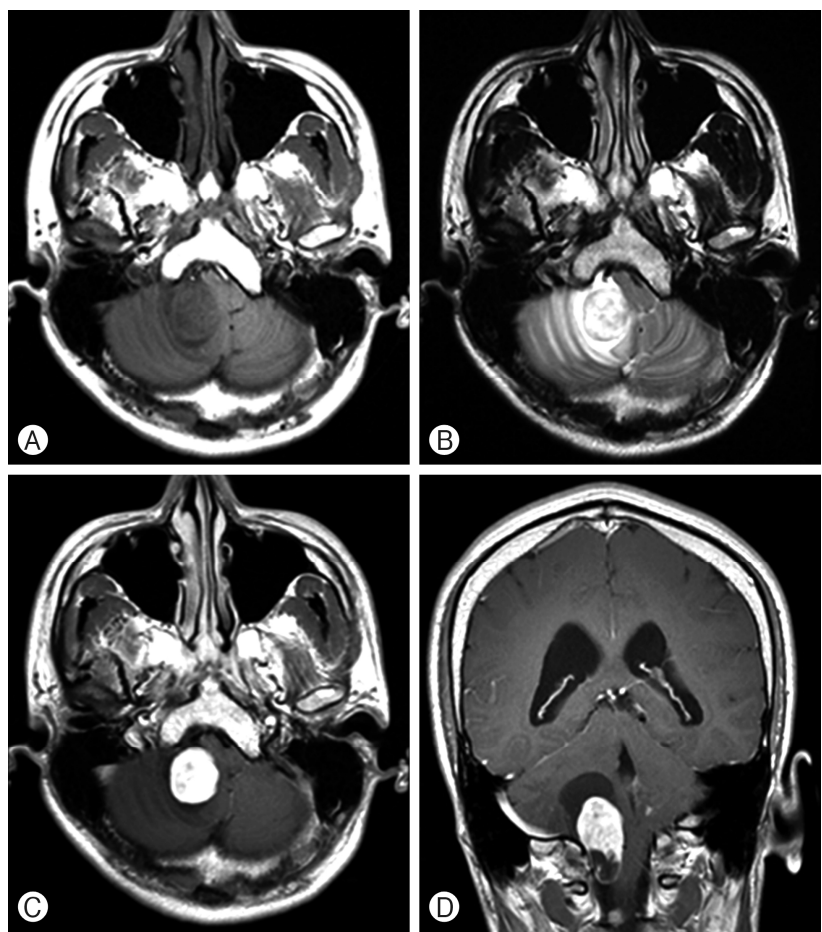

Fig. 1. (A-D) Pre-operative axial T1-weighted image (WI), axial T2$\mathrm{W}$, and contrast-enhanced axial and coronal T1-Ws showed a cystic and solid mass with avid enhancement of the solid portion in the right cerebellum.
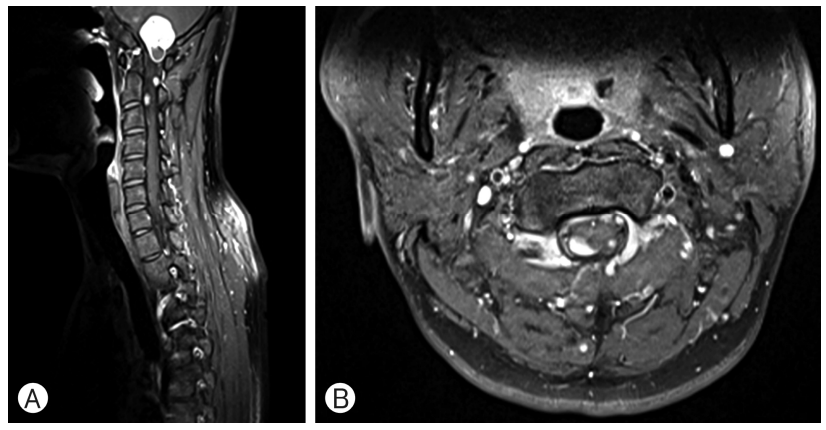

Fig. 2. (A, B) Whole-spine magnetic resonance image scan showed multiple well-enhanced intradural extramedullary nodular lesions involving $\mathrm{Cl}$ to $\mathrm{C} 3$ level.
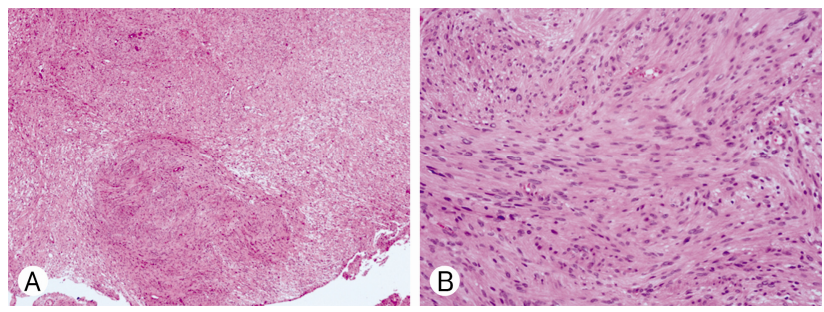

Fig. 3. (A, B) Photomicrograph showing spindle-shaped cells with elongated nuclei arranged in interlacing fascicles (hematoxylin and eosin stain $[H \& E], \times 40$ magnification; $\times 200$ magnification).

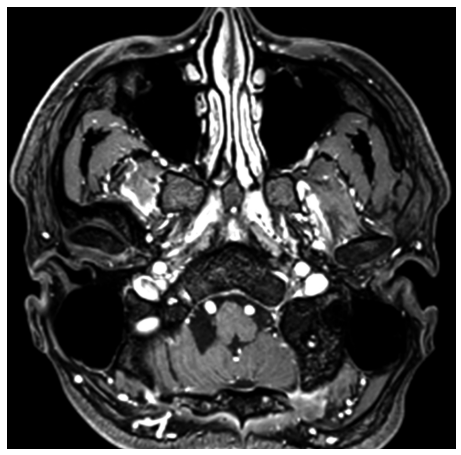

Fig. 4. One-year post-operative contrast-enhanced axial T1-weighted image showed no residual tumor and that the resection was complete.

solved by the time of discharge. The tumor was diagnosed as a schwannoma (Fig. 3); hence, he was thought to have schwannomatosis. Microscopic examination revealed that the tissues were formed by spindle cells with elongated nuclei and fibrillated cytoplasm. However, due to financial constraints, molecular analysis could not be conducted. He has been followed up for more than 1 year and his condition has been stable so far. Follow-up brain MRI has revealed no recurrence (Fig. 4).

\section{DISCUSSION}

Intraparenchymal schwannomas are very rare and some cases of intracerebellar schwannomas have been reported previously. We found that in less than 20 cases, the schwannoma was located in the cerebellar hemisphere ${ }^{5)}$. However, confirmed schwannoma within the cerebellum in presumed schwannomatosis is rare. In the case presented here, the diagnosis of schwannomatosis could not be confirmed because the patient was not tested for the $N F$ mutation due to financial constraints, but schwannomatosis was very likely because he had all the other features consistent with a diagnosis of schwannomatosis. Until recently, there have been no reports of confirmed intracerebellar schwannoma in patients with schwannomatosis.

The mechanism underlying the development of intraparenchymal schwannomas remains unknown; however, several theories have been proposed. Prakash et al. ${ }^{6}$ stated that myelinated fibers with Schwann cells may be incorporated within the substance of the brain as a hamartomatous malformation, thus forming a nidus for the growth of an intraparenchymal schwannoma. Feigin and Ogata ${ }^{3)}$ suggested that Schwann cells may originate from the differentiation of multipotential mesenchymal elements within the central nervous system.

Intracerebellar schwannomas are very rare tumors; however, they should be considered as differential diagnoses when a well-circumscribed nodule in the cerebellar hemisphere with typical signs of schwannomas is present in patients with schwannomatosis.

\section{CONCLUSION}

To our knowledge, this case report is the first to describe 
an intracerebellar schwannoma in presumed schwannomatosis. This indicates that intracerebellar schwannoma should be considered as a differential diagnoses when a nodule in the cerebellar hemisphere in patients with schwannomatosis.

\section{CONFLICTS OF INTEREST}

No potential conflict of interest relevant to this article was reported.

\section{REFERENCES}

1. Carter JM, O'Hara C, Dundas G, Gilchrist D, Collins MS, Eaton $\mathrm{K}$, et al.: Epithelioid malignant peripheral nerve sheath tumor arising in a schwannoma, in a patient with "neuroblastoma-like" schwannomatosis and a novel germline SMARCB1 mutation. Am J Surg Pathol 36:154-160, 2012

2. Casadei GP, Komori T, Scheithauer BW, Miller GM, Parisi JE, Kelly PJ: Intracranial parenchymal schwannoma. A clinicopatho- logical and neuroimaging study of nine cases. J Neurosurg 79: 217-222, 1993

3. Feigin I, Ogata J: Schwann cells and peripheral myelin within human central nervous tissues: the mesenchymal character of Schwann cells. J Neuropathol Exp Neurol 30:603-612, 1971

4. MacCollin M, Chiocca EA, Evans DG, Friedman JM, Horvitz R, Jaramillo D, et al.: Diagnostic criteria for schwannomatosis. Neurology 64:1838-1845, 2005

5. Maiuri F, Colella G, D'Acunzi G, De Caro Mdel B: Malignant intracerebellar schwannoma. J Neurooncol 66:191-195, 2004

6. Prakash B, Roy S, Tandon PN: Schwannoma of the brain stem: case report. J Neurosurg 53:121-123, 1980

7. Sarkar C, Mehta VS, Roy S: Intracerebellar schwannoma. Case report. J Neurosurg 67:120-123, 1987

8. Sharma MC, Karak AK, Gaikwad SB, Mahapatra AK, Mehta VS, Sudha K: Intracranial intraparenchymal schwannomas: a series of eight cases. J Neurol Neurosurg Psychiatry 60:200-203, 1996

9. Singh RV, Suys S, Campbell DA, Broome JC: Malignant schwannoma of the cerebellum: case report. Surg Neurol 39:128-132, 1993 